\title{
Identification and categorization in visual search
}

\author{
MURRAY J. WHITE \\ Victoria University of Wellington, Wellington, New Zealand
}

\begin{abstract}
Seven experiments were addressed to the general question of whether the identification of letters and numbers is a more rapid process than the categorization of such stimuli. Subjects were required to make a single response if a target stimulus specified by name (e.g., "A, " "2") or designated by category class alone (e.g., "letter," "number") was presented in a trial. The principal findings were: (1) identification reaction times (RTs) were faster than categorization R'Ts; (2) R'Ts for targets shown without a context were faster than RTs for targets shown in the context of other stimuli; (3) identification RTs for targets shown in the context of stimuli from a different conceptual-taxonomic category were faster than RTs for targets shown in the context of stimuli from the same category only when target-context stimulus discriminability differences were optimized. The results were interpreted in terms of a two-stage processing model in which context factors affect the duration of an initial encoding-scanning stage and search instruction (effective memory size) factors affect the duration of the memory comparison stage.
\end{abstract}

This research is concerned with the question of whether letters and numbers can be categorized faster than they can be identified by name. Operationally, categorization and identification search behaviors can be defined in terms of the response made by an observer to a particular search instruction. Consider a situation in which a number of trials each consists of the brief presentation of a single random letter or number. (1) The subject may be asked to name the letter or number shown in each trial; alternatively, he may be asked to name the superordinate category to which the stimulus belongs. In the first case, the subject is required to identify the stimulus; in the second case, he is required to categorize the stimulus. (2) The subject may be instructed to make a positive (manual) response whenever the stimulus shown in a trial matches the name of a stimulus previously specified by the experimenter; alternatively, the subject may be instructed to respond if the stimulus shown in a trial is a member of a superordinate category (letter or number) specified by the experimenter before each trial. Again, in the one case, the subject is required to respond to the name-identification of the target and, in the other, to respond to the category to which the stimulus belongs.

If identification and categorization are defined in this way, a straightforward feature-analysis model of visual memory search might predict that identification

This research was supported in part by grants from Victoria University of Wellington (62/76) and the University Grants Committee $(70 / 51)$. The critical advice of Geoff White is gratefully acknowledged, as is the assistance of Julie Marwick, Anthony McLean, Shirley Hutchison, Guy Jenner, Kim Skinner, and Miriam Williams, in collecting data for Experiments 3,5, and 6. decision latencies will be faster than categorization decision latencies. Suppose the observer is asked to respond only if the forthcoming stimulus is " 4 ." The subject sets up a memory respresentation of the stimulus " 4 " and, during some time after stimulus onset, compares this representation with the encoded representation of the distal target. A similar comparison process might occur with instructions to respond if the forthcoming stimulus is a number, only then, the memory set size will necessarily consist of a larger pool of items.

Consider a different experimental situation, wherein the subject is asked to discriminate a target identified by the experimenter, presented in the context of other stimuli. Search times will be faster when stimulus discriminability differences are maximized (e.g., searching for a $Z$ in rows composed of the letters $\mathrm{O}, \mathrm{D}, \mathrm{U}, \mathrm{G}, \mathrm{Q}$, and $\mathrm{C}$ ) than when they are minimized (e.g., searching for a $Z$ in rows composed of the letters I, V, M, X, E, and N) (cf. Neisser, 1963, 1967). This finding is readily accommodated by a feature-analysis model of visual search. Not so well accommodated is the finding that visual search is facilitated when the target and context stimuli belong to different conceptual categories. For example, the letter $Z$ is discriminated more rapidly when shown in rows composed of random numbers than when it is shown in rows composed of other letters (e.g., Brand, 1971; Ingling, 1972). Returning to the general question of identification and categorization search behaviors, visual search context effects have been interpreted in terms of models which require stimulus categorization to precede, or use less information than, stimulus identification (e.g., Ingling, 1972).

The question of whether the categorization of letters and numbers preempts the identification of such stimuli 
is not clearly answered by existing research. To the contrary, the question is confused by procedural and interpretative problems. Thus, one might argue that identification precedes categorization because decision latencies with name-identification instructions are faster than latencies found with categorization instructions (e.g., Dick, 1971). One might equally argue that categorization precedes identification because search latencies are faster for a target presented in the context of stimuli from a different conceptual category than for a target presented in the context of stimuli from the same conceptual category (e.g., Brand, 1971; Ingling, 1972; Jonides \& Gleitman, 1972, 1976).

The present paper first reviews the experimental evidence relating to the identification and categorization of letters and numbers, noting the procedural and interpretative problems in this research. This review will furnish a number of questions, the answers to which will be sought in a series of visual (tachistoscopic) search experiments.

In the classical visual search paradigm, subjects discriminate a letter embedded in rows composed of numbers more rapidly than they discriminate a letter embedded in letter rows; a number shown in rows of letters is discriminated more rapidly than a number shown in rows of numbers (Brand, 1971; Ingling, 1972; Sperling, Budiansky, Spivak, \& Johnson, 1971). In the tachistoscopic search paradigm, subjects discriminate a specified letter shown in an array of numbers faster than they discriminate a letter shown in an array of other letters (Gleitman \& Jonides, 1976; Jonides \& Gleitman, 1972, 1976).

"Category" effects have been found in other experimental paradigms. When subjects are asked to identify a target letter embedded in a row of other letters, backward masking impairs the identification of a target letter occupying middle-of-row (foveal) positions, but not a target occupying end-of-row (peripheral) positions. No selective masking effects are found when the target is a number and the contextual stimuli are letters (Butler, 1975). Using meaningful word stimuli, Karlin and Bower (1976) have shown that search reaction times (RTs) are faster for a target word shown in the context of words from a different semantic category than for a target word shown in the context of stimuli from the same semantic category.

The most appealing theoretical explanation for category effects in visual search seems to be that conceptual categorization occurs before, or requires less information than, stimulus identification. This notion is central to the explanations proposed by Butler (1975), Ingling (1972), and Jonides and Gleitman (1976). Thus, Ingling writes, "encoding information by category at an early stage in perceptual processing without first making a more specific identification can increase the rate of processing in comparison with the rate of processing more specific identification. Information which identifies symbols is not transmitted when such a savings in processing time occurs" (1972, p. 240). Butler (1975) has interpreted the selective backward masking results in a similar way, proposing a two-stage processing model in which "the directable component for attention must be preceded by a stage of processing in which the category, but not the identity of each item, is established" (p. 475).

The "partial processing" hypothesis advanced by Jonides and Gleitman (1976) suggests that "categorization (e.g., deciding whether an array item is a digit) requires less detailed processing than does identification (e.g., deciding whether an array item is a 4), thus resulting in less examination time for each item presented and hence a faster reaction time for between- than for within-category search" (p. 297). Such a hypothesis predicts, for example, that less information about the context items is remembered in the between- than in the within-categories search condition. The hypothesis also predicts that less information is required to determine the category of an item. Both these predictions have been experimentally verified (Gleitman \& Jonides, 1976; Jonides \& Gleitman, 1976).

While there is, therefore, a substantial amount of empirical evidence supporting the idea that categorization preempts identification, the notion is not uniformly watertight. Three difficulties may be cited. First, it has been found that subjects are able to search as rapidly for an unspecified target (identified only by category) as for a specified target (identified by name) (e.g., Brand, 1971; Sperling et al., 1971), and while this has been treated as independent support for a categorization hypothesis, it is open to alternative interpretations. Brand (1971) observed equally fast categorization and identification search times for 5 of 12 subjects, suggesting thereby that categorization might occur without identification. The possibility that subjects adopted a uniform search and comparison strategy (any one subject searched through lists of either letters or numbers, looking for a target number or letter) is strengthened by Brand's admission that "two of the subjects who practiced the task claimed that they were in fact doing this; in scanning through letters, for example, they would set themselves to scan for a 'digit' even if told that the target was ' 3 "' (p. 184).

A second problem for a serial categorization-beforeidentification hypothesis has to do with stimulus discriminability effects. The findings of Neisser (1963, 1967), in particular, would imply that target-context stimulus-feature differences assist discrimination in the between-categories condition and hinder discrimination in the within-categories condition. In the case of letters and numbers, stimulus discriminability factors would include symmetry about the vertical 
axis (more letters than numbers are symmetrical), and stimulus width (letters are typically "fatter" than numbers). There has been only one well controlled experiment refuting the importance of stimulus discriminability effects. Jonides and Gleitman (1972) observed that the letter " $O$ " was discriminated faster when it was designated "zero" or " $\overline{0}$ " ("oh") and shown in arrays of letters or numbers, respectively (between categories), than when the same physical target was designated $\bar{o}$ or zero and shown in arrays of letters or numbers, respectively (within categories). A major difficulty in interpreting Jonides and Gleitman's results is that nowhere did they report whether category differences were statistically significant; for some array sizes these differences were clearly negligible. By way of contrast, Karlin and Bower (1976) have reported that with a memory set size of one or three words, subjects are able to discriminate a target word on the basis of its physical features without making reference to (encoding) the semantic category information provided by contextual items.

A third and more serious difficulty for a categorization hypothesis is that the supporting evidence has come from experiments in which the context factor alone has been manipulated; categorization and identification search behaviors have been inferred from a comparison of between- and within-categories search latencies. It has generally been argued that the relatively slow search rate in the within-categories condition reflects an item-by-item naming-identification process; the relatively fast search rate in the between-categories condition reflects a process whereby the subject can categorize the target without necessarily having to identify the contextual items (cf. Brand, 1971; Gleitman \& Jonides, 1976; Ingling, 1972; Jonides \& Gleitman, 1972, 1976).

In order to more positively distinguish between identification and categorization performance, it would seem desirable to manipulate and assess the effects of both conceptual context (between-within) and search instruction (identify-categorize). Two experiments have been reported in which search instruction (but not context) was manipulated. Dick (1971) and Nickerson (1973) had subjects respond with, or to, the name of a single letter or number, and respond with, or to, the category of the item. Identification performances were seen to be faster and more accurate than categorization performances. Nickerson, who varied the amount of perceptual "noise" in a stimulus display, found that it was not possible for subjects to classify the characters vis a vis the digit-letter distinction on the basis of less stimulus information than was required to identify the characters.

The present experiments are therefore concerned with resolving the following questions: (1) Can letters and numbers be identified faster than they can be categorized? (2) Is the identification-categorization process affected by whether or not the target stimulus is shown in the context of other stimuli? (3) Do the effects of relative context reflect differences in how a target stimulus is processed at a conceptual, memory comparison level, or do they reflect more, differences occurring at a stimulus-feature extraction level? Operationally, identification will be equated with a response of, or to, the subordinate name of a target stimulus; categorization will be equated with a response of, or to, the superordinate category to which the target belongs.

\section{GENERAL METHOD}

Stimulus arrays were presented in one field of a Gerbrands tachistoscope. A preexposure field showing a small centered dot which was coincident with the center of all arrays was initially illuminated for $2 \mathrm{sec}$. On the offset of this field, a stimulus array was exposed for a predetermined duration. Following offset of stimulus exposure, an illuminated postexposure field was shown for $2 \mathrm{sec}$. The luminances of all three fields were matched at $17 \mathrm{~cd} / \mathrm{m}^{2}$.

The subject was instructed to make a rapid manual response (vocal response in Experiment 1) when a target, whose name or category was specified by the experimenter before each trial, was shown in the trial. The subject pressed a telegraph key with the fingers of his/her preferred hand. In each experimental condition, half of the total number of stimulus presentations required a response and half of them did not require a response (go, no-go). The dependent variable score in all experiments was the median RT for correct responses for each subject in each treatment condition. Decision errors were found to be typically around $4 \%$ of the number of total trials and were not analyzed.

In all experiments, conditions, treatments, and stimulus presentation orders were suitably counterbalanced and/or randomized. Following each trial, the subject was informed about his/her response accuracy but not about his/her response latency. Appropriate practice trials preceded each series of experimental trials.

A total of 64 men and women served as experimental subjects. Six different subjects served in each of the Experiments 1-4; 10 different subjects served in each of Experiments 5 and 7;20 subjects served in Experiment 6. No particular subject served in more than one experiment. The subject pool consisted of students enrolled at Victoria University. Services were offered voluntarily.

\section{Search Instructions}

In order to assess how rapidly a person can respond to the category of a target stimulus, and in order that this performance might be compared with how rapidly the same stimulus can be identified, the target stimulus must be physically localized with respect to the contextual items. Consider an array of letters in which the target is $J$ (e.g., PLNKJSRF). Identification performance could be assessed from the effects of instructions to "respond if the letter $J$ is present in the array." Category performance might require assessing the effects of instructions to "respond if there is a letter in the array." Clearly, with the latter instructions, there is no cue designating the letter $J$ as the target; the subject simply has to respond to any letter seen. This will result in attenuated "categorization" latencies and make for a meaningless comparison of identification and categorization performances.

If, however, the target is cued (e.g., by positioning bar markers above and below the letter $\mathrm{J}$ ), the subject might then be instructed to "respond if the cued stimulus is a $\mathrm{J}$ " 
(identification search) and to "respond if the cued stimulus is a letter" (categorization search).

\section{EXPERIMENT 1}

This experiment was a partial replication and an extension of Dick's (1971) experiment. The extension required subjects to respond with the name or the category of a cued stimulus shown in the context of other items.

\section{Method}

No-context condition. Each of eight cards had one letter mounted in one of eight stimulus positions and each of another eight cards had one number mounted in one of eight stimulus positions. The letter and number stimuli were: $F, J, K, L, N, P$, $R, S$, and $2,3,4,5,6,7,8,9$. Letters and numbers each subtended the same vertical visual angle $(.5 \mathrm{deg})$ and varied from $.25 \mathrm{deg}$ to $.37 \mathrm{deg}$ horizontally. Stimuli occupied the following positions with respect to the fixation point: 1.75 , $1.25, .75$, and $.25 \mathrm{deg}$ to the left, and $.25, .75,1.25$, and $1.75 \mathrm{deg}$ to the right. Assignment of a letter or a number to a particular position was randomly determined, with each letter and number appearing once in the 16 cards. One thin black bar, subtending $.5 \mathrm{deg}$ vertical visual angle, was positioned above and below each target stimulus; the distance from the nearest point of a bar to the nearest point of a stimulus was also $5 \mathrm{deg}$.

Context condition. The cards in two other sets corresponded in every way to the no-context cards with the following modifications: (1) Letter within categories. On each of eight single-letter cards, the remaining seven stimulus positions were filled with a random assignment of the seven remaining letters. Letter between categories. On each of eight single-letter cards, the seven remaining positions were filled with a random assignment of seven numbers. (2) Number within categories. On each of eight single-number cards, the seven remaining positions were filled with a random assignment of the seven remaining

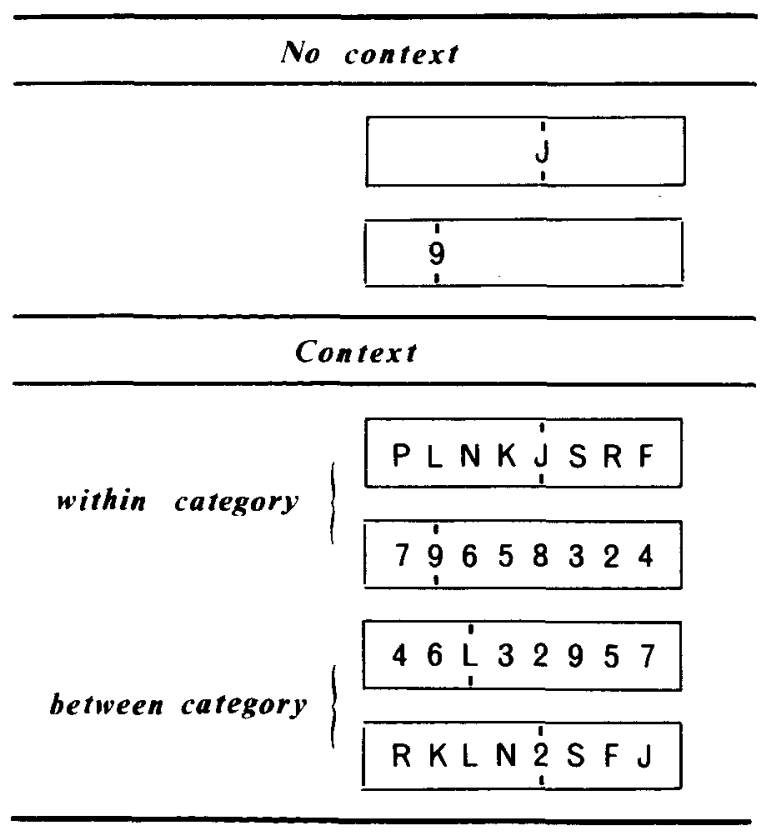

Figure 1. Examples of stimulus arrays: Experiments 1-3. numbers. Number between categories. On each of eight singlenumber cards, the seven remaining positions were filled with a random assignment of seven letters. Examples of stimulus arrays are shown in Figure 1.

There were two search instruction conditions: (1) identification, where the subject was asked to name the cued letter or number shown in each trial, and (2) categorization, where the subject was asked to name the superordinate category of the cued stimulus. The first 32 trials consisted of two randomized runs of the 16 no-context cards; the remaining 64 trials consisted of two randomized runs of the 32 context cards. Stimulus exposure was 500 msec.

\section{Results and Discussion}

Two independent ANOVAs were performed, one on the correct RTs from the no-context condition and one on the correct RTs from the context condition. In the no-context condition, the mean identification RT was $538 \mathrm{msec}$ and the mean categorization RT was $628 \mathrm{msec}[F(1,5)=9.94, p<.05]$. There were no other effects and no response errors. In the context condition, the mean identification RT was $712 \mathrm{msec}$ and the mean categorization RT was $784 \mathrm{msec}$ $[F(1,5)=13.17, p<.025]$. There were no context effects and no letter-number effects. The mean response error rate was $2 \%$.

This experiment confirms the findings of Dick (1971) and extends his observation of faster identification RTs to letters and numbers shown in the context of other letters and numbers. One explanation for these findings is, of course, that it took longer for subjects to organize the two-syllable responses "letter" and "number" than it did to organize the monosyllables "K," "4," and so on (Nickerson, 1973). Experiment 2 replicated the procedure of Experiment 1, substituting a manual go/no-go response for the spoken response required in Experiment 1.

\section{EXPERIMENT 2}

\section{Method}

An additional set of 48 no-response cards was composed, corresponding to the format of the cards used in Experiment 1 . The first session consisted of two runs of the 32 no-context cards, and the second and third sessions consisted of two runs of the 62 context cards. In all runs, half of the trials required a response and half did not. In no-response trials, the subject was told to respond if the target stimulus was a letter or a number (or, for example, if the stimulus was an " $L$ " or a "4"); he/she was then shown a card in which the cued stimulus was a number or a letter, respectively. Subjects were instructed to press the key rapidly, only when (l) the letter or number named by the experimenter was visually presented as the cued letter or number (identification search), or (2) the stimulus category "letter" or "number" spoken by the experimenter corresponded to the category of the cued stimulus (categorization search).

\section{Results and Discussion}

In the no-context condition, the mean identification RT (453 $\mathrm{msec}$ ) was faster than the mean categorization RT $(518 \mathrm{msec})[\mathrm{F}(1,5)=10.48, \mathrm{p}<.025]$. The mean error rate was $2.6 \%$. In the context condition, only 
the search instruction variable reached significance $[F(1,5)=27.50, p<.005]$. The mean identification RT was $568 \mathrm{msec}$ and the mean categorization RT was $662 \mathrm{msec}$. The mean error rate was $1.8 \%$. The similarity between these results and those found in Experiment 1 argues against response initiation (Nickerson, 1973) and implicit speech (Eriksen, Pollack, \& Montague, 1970) explanations for the faster identification search times reported by Dick (1971).

\section{EXPERIMENT 3}

Nickerson (1973) has also suggested that differences in stimulus inspection time might account for the differences between the results observed by himself and by Dick (1971), on the one hand, and by Brand (1971) and Ingling (1972), on the other. Experiments supporting a categorization hypothesis have, in fact, had subjects working under extreme time pressure or have used fast stimulus exposures ( $<200 \mathrm{msec}$ ). Dick and Nickerson exposed their single-letter and number stimuli for $400 \mathrm{msec}$ and $1 \mathrm{sec}$, respectively. In Experiment 3, three stimulus durations were employed: $100 \mathrm{msec}, 250 \mathrm{msec}$, and $500 \mathrm{msec}$.

\section{Method}

Stimuli were the same as in Experiment 2. Six subjects each served in nine sessions. In the first three sessions, subjects were asked to respond to the category or to the name of a target stimulus shown without a context. In the fourth to ninth sessions, subjects were required to categorize or to identify a stimulus shown in context. Exposure duration and search instruction variables were counterbalanced between and within sessions.

\section{Results and Discussion}

In the no-context condition, identification RTs were faster than categorization RTs (376 and $413 \mathrm{msec}$, respectively) $[F(1,5)=18.72, p<.01]$. There were no effects due to the letter-number and exposure duration variables. Mean error rates were $.2 \%$ misses and $2.1 \%$ false alarms. In the context condition, identification RTs were again faster than categorization RTs (467 and $533 \mathrm{msec}$, respectively) $[\mathrm{F}(1,5)=98.04$, $\mathrm{p}<.001]$. There was also a significant interaction between search instruction and category $[F(1,5)=10.19$, $p<.025]$. This interaction showed identification responses were faster in the between- than in the withincategories condition $[\mathrm{t}(5)=3.06, \mathrm{p}<.05]$. There was no category effect for categorization responses $[t(5)=2.08]$, due to the very large range among between-within difference RTs ( $459 \mathrm{msec}$ with $\mathrm{M}=34$; see Table 2). No other effects were statistically significant. The mean miss rate was $3.6 \%$ and the mean false alarm rate, $4.6 \%$.

The observation that identification search times were faster than categorization search times suggests that the results of Dick (1971) and Nickerson (1973) cannot be easily harmonized with those of Brand (1971) and Ingling (1972) in terms of stimulus study-time differences. The faster identification RTs for the between-categories condition can, perhaps, be attributed to the increasing importance of target-context stimulus discriminability effects with increased practice. In Experiments 1 and 2, where subjects served in only one or two context sessions, there were no Search Instruction by Category interactions. In Experiment 3, each subject served in six consecutive context sessions. In this experiment, the mean withinbetween RT difference for the results from Sessions 1 and 2 combined was $7 \mathrm{msec}[\mathrm{t}(5)<1]$. The mean RT difference for Sessions 5 and 6 combined was $29 \mathrm{msec}$ $[\mathrm{t}(5)=4.22, \mathrm{p}<.01]$. The interpretation that with increased practice, subjects increasingly utilize stimulusfeature differences in order to make a more rapid identification (even when the target is physically localized) is supported by the results of Yonas (cited in Sternberg, 1969, p. 439).

\section{EXPERIMENT 4}

In Experiments 1-3, target stimuli were physically localized by bar markers. This was necessary so that search instruction could be manipulated in both context and no-context conditions. One objection to this procedure is that the bar markers allow subjects to selectively attend to the target stimulus without "reference" to the surrounding contextual elements (cf. Jonides \& Gleitman, 1976). While this objection is not sustained by the Search Instruction by Category interaction found in Experiment 3, a further experiment was conducted in which the bar markers were omitted from displays.

\section{Method}

Stimuli consisted of the 64 context cards used in Experiment 2, minus the bar markers. Each of six subjects served in two sessions of 64 trials. The instructions in one session were to respond whenever a letter or a number named by the experimenter before each trial was shown in the following stimulus exposure. The instructions in the other session were to respond if any letter or number was shown in a trial, the experimenter having previously called "letter" or "number." Stimulus exposure duration was $500 \mathrm{msec}$.

\section{Results and Discussion}

The Instruction by Category interaction was again significant $[F(1,5)=20.51, p<.01]$. The mean categorization search time in the within-categories condition was $492 \mathrm{msec}$, and in the between-categories condition, $644 \mathrm{msec}[\mathrm{t}(5)=5.61, \mathrm{p}<.005]$. The mean identification search time in the within-categories condition was $614 \mathrm{msec}$, and in the between-categories condition, $556 \mathrm{msec}[\mathrm{t}(5)=2.59, \mathrm{p}<.05]$. The only other reliable effect showed within-categories stimuli to be discriminated faster than between-categories stimuli $[F(1,5)=11.46, p<.025]$. This result can be attributed to the discrepantly fast RT for categori- 
Table 1

Summary of Identification and Categorization Response Latencies, Experiments 1-4

\begin{tabular}{cccccc}
\hline \multirow{2}{*}{$\begin{array}{c}\text { Experj- } \\
\text { ment }\end{array}$} & \multicolumn{2}{c}{ Categorization } & & \multicolumn{2}{c}{ Identification } \\
\cline { 2 - 3 } \cline { 5 - 5 } \cline { 5 - 5 } & No-Context & Context & No-Context & Context \\
\hline 1 & 628 & 784 & & 538 & 712 \\
2 & 518 & 662 & & 453 & 568 \\
3 & 413 & 529 & & 376 & 466 \\
4 & & 568 & & 585 \\
\hline
\end{tabular}

Table 2

Summary of Search Instruction by Context Interactions, Experiments 1-4

\begin{tabular}{|c|c|c|c|c|}
\hline \multirow{2}{*}{$\begin{array}{c}\text { Experi- } \\
\text { ment }\end{array}$} & \multicolumn{2}{|c|}{ Categorization } & \multicolumn{2}{|c|}{ Identification } \\
\hline & Between & Within & Between & Within \\
\hline 1 & 796 & 772 & 714 & 710 \\
\hline 2 & 676 & 648 & 570 & 566 \\
\hline 3 & 546 & 512 & 459 & 474 \\
\hline 4 & 644 & 492 & 556 & 614 \\
\hline
\end{tabular}

zation search in the within-categories condition. Here, the subject simply had to respond to the presence of any letter or number in arrays composed solely of letters or numbers. Mean error rates were: $2.9 \%$ false alarms and $6.5 \%$ misses.

The response latency results from Experiments 14 are summarized in Tables 1 and 2 .

\section{DISCUSSION OF EXPERIMENTS 14}

The effects of specifically manipulating search instruction are clearly evident in Experiments 14 . Subjects responded to a target stimulus faster when it was identified by name than when its categorical membership alone was specified. This effect was robust, being observed (1) for target stimuli presented with and without contextual items, (2) with vocal and manual response indicators, and (3) within a range of stimulus inspection times from 100 to $500 \mathrm{msec}$. If, as Jonides and Gleitman (1976) argue, identification (operationally) involves "deciding whether an item is a 4" and categorization involves "deciding whether an array item is a digit," the present results must be seen to support a preemptive identification encoding process.

The failure to find context effects in Experiments 1 and 2 is consistent with the Jonides and Gleitman (1976) suggestion that subjects do not attend to contextual items when the target is physically localized. This suggestion must, however, be tempered by a consideration of the Search Instruction by Context interaction observed in Experiment 3. One might argue that with increased practice, subjects increasingly attend to target-context stimulus-feature relationships even when target stimuli are localized. Overall, the effects of search instruction clearly preempted or interacted strongly with the effects of context.
As was mentioned earlier, a categorization hypothesis depends on the validity of a particular interpretation of context effects. Do the effects of context on search RTs reflect conceptual, memory comparison differences or differences in physical-feature discrimination between target and contextual items? This question is addressed in Experiments 5.7.

\section{Stimulus Discriminability}

Jonides and Gleitman (1972) found that subjects discriminated the presence or absence of the stimulus letter " $O$ " faster when it was designated $\bar{o}(\mathrm{oh})$ and shown in the context of numbers than when it was designated $\overline{0}$ and shown in the context of letters. This finding underpins what might be called a conceptual encoding hypothesis, namely, that visual search RTs depend upon "how the stimulus array is conceptually categorized rather than upon its physical characteristics" (Jonides \& Gleitman, 1972, p. 457).

A second hypothesis can be proposed: Category effects reflect target-context stimulus discriminability differences. This hypothesis might naturally be called the stimulus discriminability hypothesis. Experiments 5-7 evaluate the merits of these two hypotheses. While the findings of Jonides and Gleitman would lead to the expectation of strong conceptual and weak stimulus effects, I thought it advisable to check this myself-particularly, in view of the fact that their one experiment underpins a categorization explanation for letter and number visual search RTs.

\section{EXPERIMENT 5}

Apart from the use of a within-subjects design, Experiment 5 replicated as closely as possible the sixelement present-response condition of Jonides and Gleitman's (1972) experiment. Six-element arrays were chosen because these showed the largest category RT effects in their experiment. Two predictions were tested: (1) If category effects are due to conceptual encoding differences, between-categories RTs should be faster than within-categories RTs, irrespective of the physical relationship between target and field items. (2) If category effects are due more to physical-feature differences between target and field items, the stimulus " $O$ " should be discriminated faster in the context of numbers than in the context of letters, whether it is designated $\bar{o}$ or zero. This follows from the fact that the stimulus letter $O$ is physically more similar to letters than to numbers, subtending a wider visual angle than the typical number element.

\footnotetext{
Method

Stimuli were constructed to approximate as closely as possible the six-element arrays used by Jonides and Gleitman (1972). Letters and numbers each subtended $.51 \mathrm{deg}$ in height. The mean width of number stimuli was $.29 \mathrm{deg}$, of letters, $.36 \mathrm{deg}$, and the width of the target " $O$ " was $.44 \mathrm{deg}$. Elements were mounted on the circumference of an imaginary circle
} 
whose diameter was $3.4 \mathrm{deg}$. Six stimulus elements appeared on each of 96 cards, mounted at the following clock positions: $0100,0300,0500,0700,0900$, and 1100 hours. Target letters and numbers were $O$ (the letter $O$ being used both as the $\bar{O}$ and zero targets), $A$, and 4 . The context letters included all uppercase letters, excluding $A, B, D, G, M, O, Q, W$, and $Z$; the context numbers were $1,3,5,6,8$, and 9 . Each target letter ( $O$ and $A)$ and each target number $(O$ and 4) appeared once in 12 cards and an equal number of times at each of the six clock positions. Remaining stimulus positions were filled with a random assignment of five context letters or five context numbers, making a total of eight sets (four targets by two categories) of six cards. An equal number of cards was composed in which no target letters and numbers appeared. Figure 2 shows the relationship between nontarget and target arrays. Each target card, whether between- or within-categories, had a nontarget mate in which the same context elements appeared in the same clock positions.

Three cards were randomly selected from each of the 16 subsets illustrated in Figure 2 to make two blocks of 48 . Within each block, one card from each of the 16 subsets was included in the first 16 , second 16 , and final 16 trials. Within each set of 16 trials, in both blocks, the cards were randomly ordered. Stimulus exposure was $150 \mathrm{msec}$.

\section{Results and Discussion}

The means of the median RTs are summarized in Table 3. Two separate ANOVAs were performed, one on the results for $\bar{o}$ and zero targets and one on the results for $\mathrm{A}$ and 4 targets. The first analysis showed no category and no target effects. The Category by Target interaction was significant $[F(1,9)=10.68$, $\mathrm{p}<.01]$. In the between-categories condition, the target $\overline{\mathrm{O}}$ was discriminated faster than the target zero $[\mathrm{t}(9)=2.54, \mathrm{p}<.05] . \quad$ In the within-categories condition, the target zero was discriminated faster than the target $\overline{0}[\mathrm{t}(9)=2.61, \mathrm{p}<.05]$. These results clearly

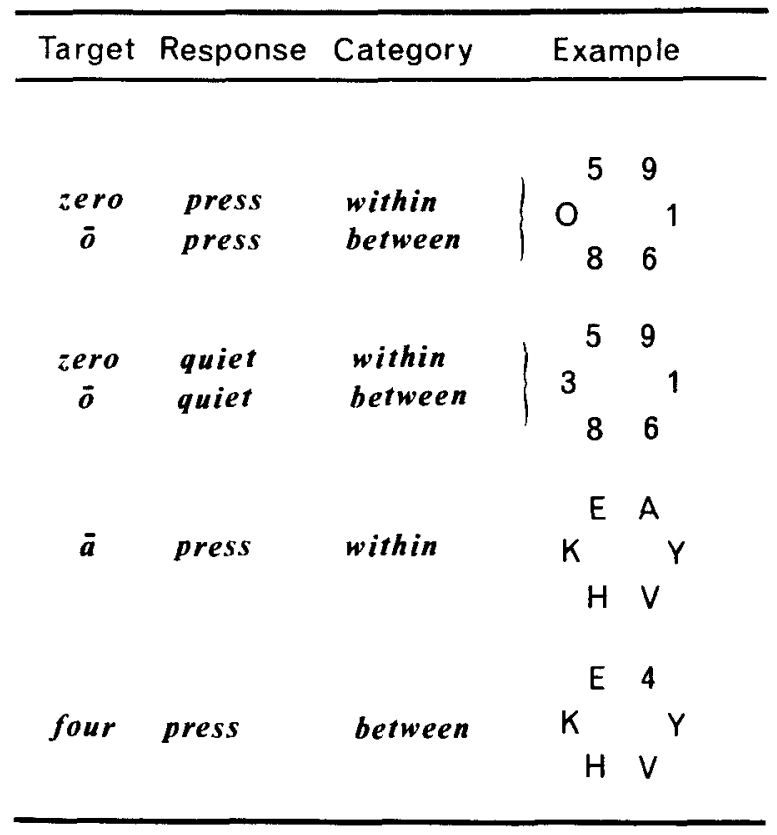

Figure 2. Examples of stimulus arrays: Experiments 5 and 6.
Table 3

Response Latencies, Experiments 5-7

\begin{tabular}{|c|c|c|c|c|c|c|}
\hline \multirow[b]{2}{*}{ Context } & \multicolumn{2}{|c|}{ Experiment 5} & \multicolumn{2}{|c|}{ Experiment 6} & \multicolumn{2}{|c|}{ Experiment 7} \\
\hline & Between & Within & Between & Within & Between & Within \\
\hline $\begin{array}{l}\overline{\mathbf{o}} \\
\text { zero }\end{array}$ & $\begin{array}{l}421 \\
434\end{array}$ & $\begin{array}{l}433 \\
414\end{array}$ & $\begin{array}{l}449 \\
474\end{array}$ & $\begin{array}{l}469 \\
442\end{array}$ & $\begin{array}{l}488 \\
482\end{array}$ & $\begin{array}{l}504 \\
480\end{array}$ \\
\hline $\begin{array}{l}A \\
4(2)\end{array}$ & $\begin{array}{l}469 \\
506\end{array}$ & $\begin{array}{l}478 \\
474\end{array}$ & $\begin{array}{l}436 \\
482\end{array}$ & $\begin{array}{l}554 \\
537\end{array}$ & $\begin{array}{l}544 \\
522\end{array}$ & $\begin{array}{l}537 \\
592\end{array}$ \\
\hline
\end{tabular}

favor a stimulus discriminability hypothesis. The second analysis, for $\mathrm{A}$ and 4 targets, showed no significant effects. The mean miss rate was $1.5 \%$; there were no false alarms.

\section{EXPERIMENT 6}

If the conceptual category effect reported by Jonides and Gleitman (1972) is robust, it would seem reasonable to expect this to have manifested itself in a withinsubjects design (Experiment 5), because this type of design minimizes subject RT variability. It is possible, however, that subjects adopt different search strategies (1) when they are able to predict the target-context format of forthcoming trials, and (2) when they are unable to make such predictions (cf. Brand, 1971). Experiment 6 was conducted in order to preserve a measure of internal consistency in making a comparison of search RTs from between- and within-subjects designs.

\section{Method}

The stimulus cards and procedure were identical to those in Experiment 5 except for the following modifications. (1) The 96 experimental cards were broken into four packs of 24 , corresponding to the following target-context relationships: (a) designated letter target in letters context, (b) designated letter target in numbers context, (c) designated number target in letters context, and (d) designated number target in numbers context. (2) The cards in each of these four subsets were presented to each of five different subjects.

\section{Results and Discussion}

The results for the $\overline{0}$ and zero targets were again analyzed separately from those for the $\mathrm{A}$ and 4 targets. These are summarized in Table 3 . For $\bar{o}$ and zero targets, there were no significant effects. There was, however, a trend more or less identical to the one observed in Experiment 5, showing the $\overline{0}$ target to be discriminated faster than the zero target in the betweencategories condition, and the zero target to be discriminated more rapidly than the $\overline{0}$ target in the within-categories condition. That this trend failed to reach an acceptable level of significance can be attributed to between-subjects RT variability. Analysis of the $A$ and 4 results showed search times were faster for between-categories search than for within-categories search $[F(1,16)=5.02, p<.05]$. The mean miss rates were $.4 \%$ ( $\overline{\mathrm{O}}$ and zero) and $1.7 \%(\mathrm{~A}$ and 4$)$. The mean 
false alarm rates were $2.5 \%$ and $8.8 \%$, respectively. In effect, the results of Experiment 6 offer little discriminative evidence for or against either hypothesis for context effects. On the one hand, the results for $A$ and 4 targets were predicted by both conceptual categorization and stimulus discriminability hypotheses. On the other hand, it is clearly not proper to argue anything definite from the null category effect observed for $\bar{o}$ and zero targets.

\section{EXPERIMENT 7}

Experiment 7 offers a more powerful test between the hypotheses. Each element in a six-element array was printed in a different typeface or typesize, so the subject could not attend to and discriminate the target on the basis of stimulus-feature differences between target and field items. If visual search RTs depend on how the array is conceptually categorized, betweencategories RTs should be faster than within-categories RTs. If context effects are determined at the level of stimulus-feature comparisons, between- and withincategories RTs should be equally fast.

\section{Method}

Stimuli were constructed in the same manner as those used in Experiments 5 and 6, with the following modifications: (1) The target 2 was substituted for 4 in order to increase discriminability between targets. (2) Context letters and numbers were $B, C, F, I, G, H$, and $3,4,6,7,8,9$. (3) Targets at each of the six positions within each of the eight possible target-context subsets were drawn in a different typeface. Fach of the tive field items in each array was also drawn in a different typeface. In each array, therefore, each element was drawn in one of six different types (see Figure 3 ). The procedure was the same as that used in Experiment 5; that is, a withinsubjects design was employed.

\section{Results and Discussion}

The RT results are summarized in Table 3 . Analysis of the $\bar{o}$ and zero targets showed no category effect and no Target by Category interaction. Response latencies for targets designated zero were faster than latencies for targets designated $\overline{\mathrm{o}}[\mathrm{F}(1,9)=15.82$, $p<.01]$. There is no obvious explanation for this. It is possible that it was due to subtle differences in rehearsing the terms $\bar{o}$ and zero; possibly $\bar{o}$ was sometimes confused with $\overline{\mathrm{a}}$ (an alternative target) in any covert rehearsal which occurred between target specification and stimulus exposure. Analysis of the A and 2 results showed no effects. The mean miss rates were $.8 \%$ for $\overline{0}$ and zero targets and $7.5 \%$ for $\mathrm{A}$ and 2 targets. The mean false alarm rates were $2.9 \%$ and $2.5 \%$, respectively.

\section{DISCUSSION OF EXPERIMENTS 5-7}

The results of Experiments $5-7$ offer little support for a hypothesis which maintains that visual search RTs depend upon how the stimulus array is conceptually

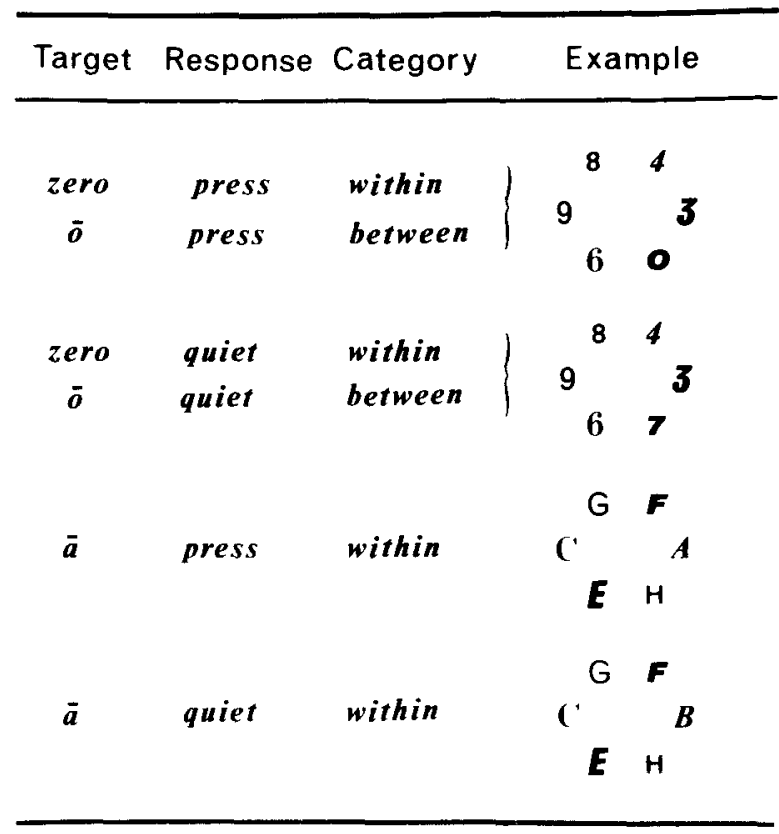

Figure 3. Examples of stimulus arrays: Experiment 7.

categorized. In Experiment 6, between-categories search RTs were faster for A and 4 than were within-categories RTs. This finding was predicted by both conceptual encoding and stimulus discriminability hypotheses. In Experiment 7, there were no category effects for A and 2, a result predicted by a stimulus discriminability hypothesis but contrary to what would be expected according to the views of Jonides and Gleitman (1972).

Of greater importance, a conceptual encoding idea would predict faster between-categories search RTs for the ambiguous target "O." This target should have been discriminated faster when designated $\bar{o}$ and shown in a numbers context or when designated zero and shown in a letters context. There was no evidence for this.

The experiments do provide reasonably strong support for the notion that letter and number context effects are determined by stimulus discriminability differences existing between target and context items. In Experiment 5, RTs to the stimulus " $O$ " were faster when it was shown in a numbers context than when it was shown in a letters context, irrespective of its designation as $\bar{o}$ or zero. The letter " $O$ " subtended a much wider visual angle than the typical number element. The nonsignificant Category by Target interaction observed in Experiment 6 showed a similar pattern of results. Finally, in Experiment 7, where physical-feature effects were controlled, search RTs for the letter " $O$ " were equally fast in both letters and numbers contexts. This was also predicted by a stimulus discriminability hypothesis.

How might the discrepancies between these results and those of Jonides and Gleitman (1972) be accounted for? One explanation might lie in the fact that in the earlier experiment, subjects viewed arrays 
of two, four, and six elements; in the present experiments, all arrays had six elements. But if conceptual category effects are sensitive to such minor procedural differences, they can hardly be called robust. Another possibility is that the category effects observed by Jonides and Gleitman were statistically nonsignificant, notwithstanding significant slope differences. However these different sets of results might be reconciled, there appears to be good reason for doubting that context effects in visual search reflect differences occurring at a conceptual, memory comparison level.

\section{GENERAL DISCUSSION}

How might the stages involved in identifying and categorizing letters and numbers be conceptualized? While there are a number of theoretical models that can accommodate different facets of the present data, the description that seems best suited overall is that proposed by Sternberg $(1969,1975)$. This is sketched in a modified form in Figure 4.

The stimulus target or array is first encoded into some, presumably visual-iconic, mental representation. The duration of this encoding stage is determined by such factors as the number of items in the visual array, stimulus discriminability differences, and the legibility

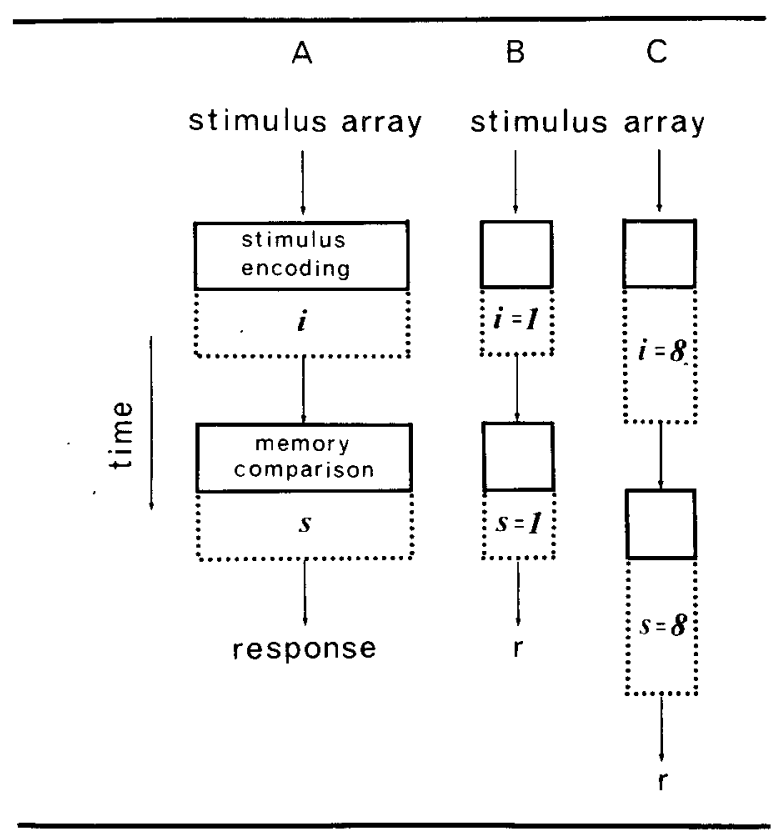

Figure 4. (A) Conceptualization of processing stages in the visual search experiment. Height of a box reflects mean duration of a particular stage. Number of array items (i) and targetcontext stimulus discriminability influence the duration of encoding and representation. Positive memory set size (s) influences the duration of the memory comparison stage. (B) Stages and hypothetical durations in the no-context identification search condition. (C) Stages and durations in the context categorization search condition. (Adapted from Sternberg, 1969, 1975.) of the array items. It is also assumed that an initial "preattentive" scan is made of the encoded array during the time the array is being encoded. Thus, a single array (target) item is encoded and focalized more rapidly than an item shown in the context of other items (Experiments 1-3). Similarly, an item that is featurally different from contextual items is encoded and focalized more rapidly than an item that is featurally similar to, or confusable with, contextual items (Experiments 5-7). The locus of context effects, absolute and relative, is thus placed at the level of stimulus encoding.

Those items that are most strongly and legibly encoded are compared with items in the memory set. The size of the positive memory set principally determines the duration of the memory comparison process. This duration will be shorter when the memory set size consists of a single item than when the memory set consists of $s>1$ items (see Figure 4). The fact that search latencies were faster with categorization instructions than with identification instructions when the target was not physically localized (Experiment 4) can be attributed to a rapid random matching of an encoded array item with any item in the positive memory set (see Table 1).

The locus of the effects of search instruction are thus placed at the memory comparison stage. The problem of serial vs. parallel search is relevant only to the process occurring with a memory set size greater than one, in other words, for the categorization search. The present experiments are not clear as to whether the comparison process proceeds serially or in parallel among items of different trace strengths. Again, primarily because negative (target-absent) responses were not required, I am unable to comment on whether the memory comparison process is self-terminating or exhaustive (cf. Sternberg, 1975). For the same reason, however, it can be argued that the positive responses reflected processes occurring before the response retrieval stage.

If it is also assumed that the two principal determinants of response time were, in the present experiments, context and search instruction (i.e., effective memory set size), then it could be expected that, as array size and/or memory set size increased, the duration of the encoding and/or memory comparison process likewise increased. Response latencies should have been fastest for the no-context (single array item) identification search (memory set size $=1$ ) condition, and should have been slowest for the context categorization condition. The mean response latencies, averaged over results from Experiments 1-3, were $456 \mathrm{msec}$ and $658 \mathrm{msec}$, respectively. Response latencies for the context identification and no-context categorization conditions should have been somewhere within this range. The mean latencies were $582 \mathrm{msec}$ and $520 \mathrm{msec}$, respectively.

The separate durations of the encoding and memory 
comparison stages cannot be assessed from these data. Nevertheless, it is clear that context had a considerable effect on encoding. Using the results from Experiments 1-3 again, the mean context/no-context RT difference with identification search was $126 \mathrm{msec}$, and with categorization search, $138 \mathrm{msec}$. By comparison, the mean categorization-identification RT difference with context arrays was $76 \mathrm{msec}$, and with no-context arrays, $64 \mathrm{msec}$. Whether these differences accurately reflect encoding/memory comparison process RT differences must be checked by a study in which memory set size and visual array size are parametrically varied.

This conceptualization of the "identificationcategorization" search process is admittedly loose. The present data do not allow for any formal or structured model; the experiments were, after all, designed to answer a number of important research questions rather than to discriminate between models of search behavior. It is clear, though, that it is neither necessary nor empirically justifiable to conceptualize a process whereby a letter or a number is recoded or translated into its category class before it is identified by name.

All this is not to say that conceptual-taxonomic category effects are necessarily determined at the level of stimulus encoding. There is evidence that for pictorial stimuli and meaningful words, visual search is facilitated by categorization (e.g., Hock, Gordon, \& Corcoran, 1976; Karlin \& Bower, 1976). There is evidence that memory search is facilitated when the positive memory set consists of items from different taxonomic categories (e.g., Naus, 1974; Naus, Glucksberg, \& Ornstein, 1972). I suggest that for these different materials and paradigms there is one or more processing stages interfacing the stages outlined in Figure 4, or, alternatively, that some additional operation is performed on the encoded items at the memory comparison stage (cf. Sternberg, 1975).

\section{REFERENCES}

Brand, J. Classification without identification in visual search. Quarterly Journal of Experimental Psychology, 1971. 23. $178-186$.
BUtleR, B. Selective attention and target search with brief visual displays. Quarterly Journal of Experimental Psychology. 1975. 27, 467.477.

Dick, A. O. Processing time for naming and categorization of letters and numbers. Perception \& Psychophysics. 1971. 9. 350-352.

Eriksen, C. W., Pollack, M. D., \& Montague, W. E. Implicit speech: Mechanism in perceptual encoding? Journal of Experimental Psychology, 1970, 84, 502-507.

Gleitman, H., \& Jonides, J. Cost of categorization in visual search: Incomplete processing of targets and field items. Perception \& Psychophysics, 1976, 20, 281-288.

Hock, H. S., Gordon, G. P., \& Corcoran, S. K. Alternative processes in the identification of familiar pictures. Memory \& Cognition. 1976. 4. 265-271.

INGLING, N. W. Categorization: A mechanism for rapid information processing. Journal of Experimental Psychology, 1972, 94, 239-243.

Jonidis. J., \& Gleitman, H. A conceptual category effect in visual search: 0 as letter or as digit. Perception \& Psychophysics, 1972. 12, 457.460.

Jonides, J.. \& Gleitman, H. Benefit of categorization in visual search: Target location without identitication. Perception \& Psychophysics, 1976, 20, 289-298.

Karlin. M. B., \& Bower, G. H. Semantic category effects in visual word search. Perception \& Psychophysics, 1976, 19. 417-424.

Naus, M. J. Memory search of categorized lists: A consideration of alternative self-terminating search strategies. Journal of Experimental Psychology. 1974. 102. $992-1000$.

Naus, M. J., Glucksberg, S., \& Ornstein, P. A. Taxonomic word categories and memory search. Cognitive Psychology, 1972, 3, 643-654.

NeIsSER. U. Decision-time without reaction-time: Experiments in visual scanning. American Journal of Psychology, 1963, 76, 376-385.

Neisser, U. Cognitive psychology. New York: AppletonCentury-Crofts, 1967.

Nickerson. R. S. Can characters be classified directly as digits vs. letters or must they be identified first? Memory \& Cognition, 1973, 1, 477-484.

Sperling, G.. Budiansky, J., Spivak, J. G., \& Johnson, M. C. Extremely rapid visual search: The maximum rate of scaming letters for the presence of a numeral. Science, 1971. 174, 307-311.

SternberG, S. Memory-scanning: Mental processes revealed by reaction-time experiments. American Scientist, 1969, 57, 421-457.

STER NBERG. S. Memory scanning: New findings and current controversies. Quarterly Journal of Experimental Psychology, 1975, 27, 1-32.

(Received for publication April 8, 1977; accepted August 26, 1977.) 